\title{
Changes in salivary oxidative status, salivary cortisol, and clinical symptoms in female patients with temporomandibular disorders during occlusal splint therapy: a 3-month follow up
}

\author{
E. Vrbanović ${ }^{1}$ I. Lapić ${ }^{2}$, D. Rogić ${ }^{2}$ and I. Z. Alajbeg ${ }^{1 *}$ (D)
}

\begin{abstract}
Background: Differences in the expression of oxidative stress (OS) markers between female patients with temporomandibular disorders (TMD) and healthy individuals indicate that OS plays a role in the pathogenesis of TMD. Because chronic exposure to stress generates oxidative damage during continuous stimulation of the hypothalamicpituitary-adrenal axis, we expected that higher levels of cortisol might be associated with higher oxidative damage. Our aim was to test the association between OS markers, stress perception, and salivary cortisol (SC) in chronic, female TMD patients. We tracked changes in OS markers and SC during occlusal splint therapy in order to evaluate the influence of treatment on oxidative status. We hypothesized that the effects of TMD therapy would differ among individuals depending on the source and intensity of pain.
\end{abstract}

Methods: Sixteen female patients were recruited, and 12 finished the study. Clinical assessment and saliva sampling were performed at the baseline and follow-up appointments. Repeated measures analysis of variance and Pearson's correlation were used for analyzing the data.

Results: After 3 months, a significant reduction in afternoon total antioxidant capacity (TAC) was observed $(p<0.05)$. A significant reduction in afternoon malondialdehyde $(\mathrm{MDA})(p=0.021)$ and a decrease in afternoon MDA to superoxide dismutase ratios $(p=0.017)$ were present in high-intensity pain patients. At baseline, higher levels of perceived stress were significantly associated with higher morning cortisol $(\rho=0.67)$. At the end of the therapy, reduced perceived stress was positively correlated with morning SC changes when considering all TMD patients, but the association between perceived stress with OS markers was present only in myofascial pain (MP) group. The effect of treatment on the selfperceived quality of life was more pronounced in female MP patients while the reduction of spontaneous pain was significantly greater in high-intensity pain patients.

Conclusion: Our data indicate that occlusal splint therapy in female TMD patients contributes to increasing their capacity to remove free radicals. The question remains whether or not TAC decreases in this process as a result of avoiding unnecessary processes, once the increase in antioxidants effectively compensates for OS. The intensity and the source of pain should be considered important factors in future investigations evaluating salivary OS markers and their association with perceived stress and SC in TMD patients.

Trial registration: ClinicalTrials.gov NCT03029494. Registered on 2017-01-19.

Keywords: Temporomandibular disorders, Oxidative stress, Salivary cortisol, Occlusal splint, Salivary diagnostics

\footnotetext{
* Correspondence: ialajbeg@sfzg.hr

1 Department of Removable Prosthodontics, School of Dental Medicine,

University of Zagreb, Gunduliceva 5, HR-10000 Zagreb, Croatia

Full list of author information is available at the end of the article
}

(c) The Author(s). 2019 Open Access This article is distributed under the terms of the Creative Commons Attribution 4.0 International License (http://creativecommons.org/licenses/by/4.0/), which permits unrestricted use, distribution, and reproduction in any medium, provided you give appropriate credit to the original author(s) and the source, provide a link to the Creative Commons license, and indicate if changes were made. The Creative Commons Public Domain Dedication waiver (http://creativecommons.org/publicdomain/zero/1.0/) applies to the data made available in this article, unless otherwise stated. 


\section{Background}

Temporomandibular disorders (TMD) encompass the most common painful musculoskeletal and neuromuscular orofacial conditions and affect jaw joints, masticatory muscles, and the surrounding structures. These conditions are still primarily diagnosed through medical history and clinical examination due to their multifactorial pathophysiology, which is still not completely clear [1]. Symptoms, signs, and behavioral risk factors are examined using well-defined, evidence-based questionnaires and protocols that allow for few diagnostic errors and are therefore considered valid methods for TMD diagnosis [2]. The majority of TMD patients are women, with the prevalence rate being the highest between 20 and 40 years of age [3]. The most common diagnoses representing pain-related TMD are myofascial pain (MP), disc displacement (DD), and degenerative joint disease [2].

The main goal of TMD therapy is the reduction of clinical symptoms such as pain and limited lower jaw movement [4]. Physical therapy, behavioral therapy, and the use of occlusal splints are often mentioned as successful treatment methods, with few or no differences in treatment outcomes [5]. In some studies, the combination of noninvasive modalities showed better results than the use of just one treatment option $[6,7]$. The role of occlusal splints is still not fully understood, but some evidence suggests that this treatment is better than no treatment at all [8]. Treatment success is considered to be due to a combination of different factors, and one of these factors is potentially a placebo effect [9].

Research has recently suggested that oxidative stress (OS) can play a part in the pathophysiological processes that occur in TMD. OS occurs as a result of an imbalance between oxidants and antioxidants in favor of oxidants, and it is believed to play a role in various adverse processes in an organism [10]. OS is being investigated as a contributing factor to various human pathologies [11-13], including those connected to oral health [14-16].

Studies have found significant differences in the expression of OS biomarkers and antioxidants between TMD patients and healthy individuals [17, 18]. Therefore, the estimation of OS biomarkers can be used as an additional tool for early detection, diagnosis, and monitoring of TMD patients. An association between higher levels of OS markers with higher pain intensity scores has been reported [17]. Our previous study addressed the differences in oxidative status by considering pain intensity and the specific diagnostic subgroups of TMD [18]. Hence, the applied therapy might depend on the clinical subtype of TMD and can have a different effect on different individuals depending on whether they report high-intensity pain (HIP) or low-intensity pain (LIP).

The connection between OS, cortisol, and psychological stress has been previously discussed [19]. Cortisol is often mentioned as an indicator of chronic stress, because heightened cortisol release occurs during the anticipation of stress [20]. The long-lasting effects of cortisol are often connected with various diseases and conditions; oxidative damage is likewise elevated in people with diseases such as neurodegenerative, metabolic, cardiovascular conditions, or cancer [21, 22]. Although increased metabolism alone generates free radicals, glucocorticoid hormones have been shown to play both direct and indirect modulatory roles in the onset of OS [23]. Because it has been suggested that chronic stress exposure generates oxidative damage during the continuous stimulation of the hypothalamicpituitary-adrenal axis [19], we expected that changes in oxidative status would be associated with changes in cortisol levels.

The aim of this study was to: 1) test the association between OS biomarkers, stress perception, and salivary cortisol (SC) in chronic TMD patients, and 2) to follow up on changes in OS markers and SC during 3 months of occlusal splint therapy in order to evaluate the influence of treatment on oxidative status. Our hypothesis was that there would be a positive association between SC, perceived stress, and OS biomarkers. We also hypothesized that the effects of TMD therapy on salivary oxidative status would differ between individuals with HIP and LIP as well as between MP and DD patients.

\section{Methods}

This study was performed at the Department of Removable Prosthodontics, School of Dental Medicine, University of Zagreb with the approval of the Ethics Committee (01-PA-26-6/15, item 3.2) and regulated in accordance with the Helsinki Declaration. All subjects were informed of the procedures involved in the study and provided with written consent before being included in the research.

\section{Participants}

A statistical power analysis was performed for sample size estimation based on data from Rodríguez de Sotillo et al. [17] which compared OS (as measured by biomarkers in saliva) in female TMD patients with high and low pain intensity. The minimum difference in salivary total antioxidant capacity (TAC) and malondialdehyde (MDA) level means was estimated to be $0.3 \mathrm{nmol} / \mathrm{L}$ and $0.49 \mathrm{pg} / \mathrm{ml}$, respectively, and the standard deviation of TAC and MDA was expected to be $0.15 \mathrm{nmol} / \mathrm{L}$ and $0.25 \mathrm{pg} / \mathrm{ml}$, respectively. With an alpha $=.05$ and power set at $80 \%$, the projected sample size was approximately $N=12$ (6 per group).

Participants were diagnosed according to the diagnostic criteria for TMD (DC/TMD) [2]. The inclusion criteria were a report of chronic pain lasting more than 6 months and a diagnosis of MP or DD. Only women 
were selected for the study because of the high prevalence of TMD in female population. The exclusion criteria were orofacial pain unrelated to TMD, degenerative joint disease, smoking, gingivitis, periodontitis, oral lesions, chronic systemic diseases (e.g., diabetes, cardiovascular diseases, cancer, and autoimmune diseases), use of supplements or medications known to affect oxidative status, and pregnancy. Patients displaying combined MP and DD diagnoses and patients who had received other TMD treatments in the preceding 6 months were also excluded. A clinician (IZA) with expertise in TMD diagnostics conducted a clinical examination of the patients.

Sixteen female patients met the inclusion criteria. During recruitment, 1 of them refused to take part in the study due to travelling complications; thus, 15 patients were enrolled in the study, but 3 patients dropped out before the salivary measurements were performed. Ultimately, 12 patients had all of the required measurements performed at the baseline and follow-up appointments (Fig. 1).

\section{Grouping of patients}

First, patients were grouped according to their source of pain in order to explore the interactions of OS and pain sources, and two groups were formed (6 MP and $6 \mathrm{DD})$.

Next, the patients were grouped according to pain intensity as measured by the characteristic pain intensity (CPI) using the graded chronic pain scale [2]. To calculate $\mathrm{CPI}$, the means of current pain, worst pain, and average pain are computed and multiplied by $10(\mathrm{CPI}<50$ represents LIP and CPI $\geq 50$ represents HIP). Six patients were labeled as having HIP and six as having LIP.

\section{Treatment protocol}

All patients received a hard acrylic (Resilit-S, Erkodent, Siemensstraße 3, 72285 Pfalzgrafenweiler, Germany) occlusal splint (stabilization splint) to wear on the maxillary dental arch. The splint was made using a stone cast in an ARTEX articulator (Amann Girrbach, Austria) in the referent position of the centric relation, with a thickness of $1.5 \mathrm{~mm}$ at the level of the first molar. The same dental technician made all of the splints. A clinician (IZA) adjusted the splints so that opposing teeth occluded simultaneously with the splint surface. The same clinician adapted the splints as necessary at follow-up appointments.

\section{Outcome measures}

The study followed changes in spontaneous pain as assessed by a visual analogue scale (VAS), maximal comfortable mouth opening (MCO), the Oral Health Impact Profile (OHIP-14), and the level of perceived stress (Perceived Stress Scale [PSS]).

\section{VAS pain}

A $100 \mathrm{~mm}$ horizontal VAS scale was used to evaluate spontaneous pain from the temporomandibular joint and the masticatory muscles. The range on the scale went from "no pain" $(0 \mathrm{~mm})$ to "worst pain imaginable" (100 $\mathrm{mm})$ [24]. Only subjects with spontaneous pain of $>30$ $\mathrm{mm}$ on the VAS were included in the study.

\section{MCO}

MCO was measured as the distance between the maxillary and mandibular central incisors. A pain-free opening was defined as the maximum distance the participant could

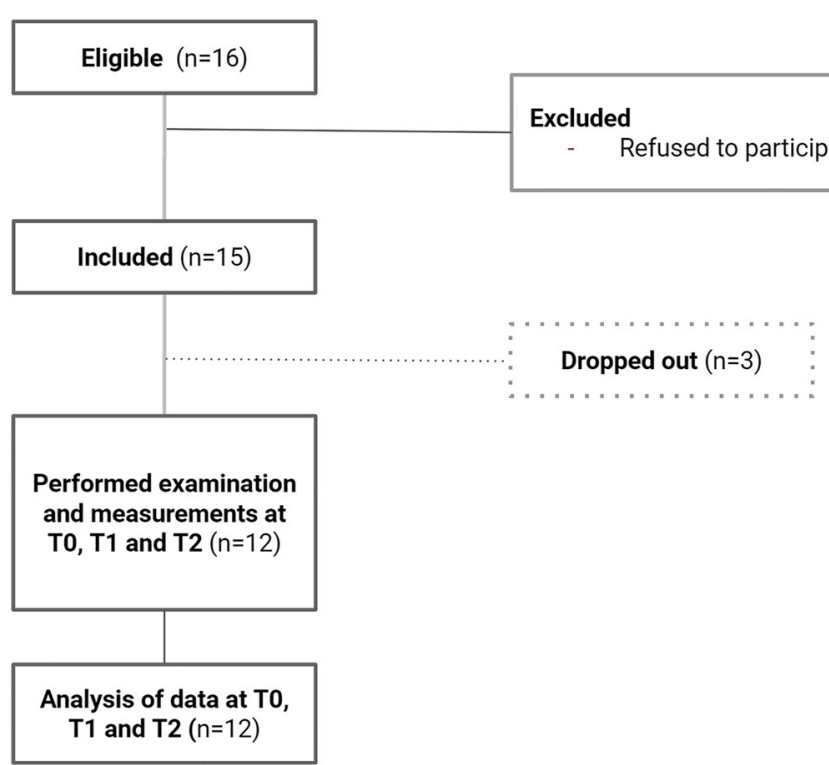

Fig. 1 Flow diagram of the progress through the phases of the study 
open her mouth without experiencing any additional pain or discomfort [7].

\section{PSS}

The PSS is a standard 10-item questionnaire that assesses subjective perceptions of stress over the previous month. Response options form a 5-point Likert scale: 0 = never, 1 = almost never, 2 = sometimes, 3 = fairly often, $4=$ very often. Possible scores ranged from 0 to 40 , with higher numbers indicating higher levels of stress. The questionnaire was previously translated into and validated in Croatian by Hudek-Knežević et al. [25].

\section{OHIP-14}

The short-form OHIP-14 questionnaire [26], which has been translated into and validated in Croatian, was used to show how oral outcomes (TMD-related pain and disability) impact patients' quality of life. Patients expressed their status through 14 questions, choosing 1 of the 5 possible answers: $0=$ never, 1 = hardly ever, 2 = sometimes, 3 = fairly often, and $4=$ very often. Possible scores ranged from 0 to 56 . The instrument was previously validated for the evaluation of TMD patients [27].

A clinician (EV) performed a clinical examination of the patients at the baseline and follow-up appointments at the first and third months after the initiation of treatment; the clinician was blinded as to each patient's CPI and specific diagnostic subgroup.

\section{Saliva collection}

Five $\mathrm{mL}$ whole, unstimulated saliva samples were collected in graduated tubes $(50 \mathrm{~mL}$ self-standing centrifuge tubes, Ratiolab, Germany). Saliva aliquots $(1 \mathrm{~mL})$ were stored at $-80^{\circ} \mathrm{C}$ until the analysis. Some of the markers show significant diurnal variations; therefore, the saliva was collected in the morning (7 AM) and in the afternoon (5 PM) [16].

Morning sampling was performed at home before tooth brushing. Subjects were instructed to fast before saliva collection in the morning and to not eat or drink anything but water for at least 2 hours before sampling in the afternoon. All saliva samples were collected after rinsing the mouth with water. Brushing teeth before saliva sampling was forbidden to avoid blood contamination. Saliva was collected at follow-up measurements at the first and third months of occlusal splint therapy, following the same procedure. The sample collection and storage principles used in the study are described in detail by Alajbeg et al. [28].

\section{Salivary analysis of MDA, TAC, superoxide dismutase (SOD), and uric acid (UA)}

The sample analysis was performed at the Department of Laboratory Diagnostics, University Hospital Center, Zagreb.
The following OS biomarkers were analyzed: MDA, TAC, SOD, and UA.

MDA was determined using the MDA Adduct Competitive ELISA kit (Kamiya Biomedical Company, Seattle, USA), which measures the MDA-stable hybrid protein adducts (MDA adducts), referred to as lipid peroxidation end products, formed by binding MDA to proteins.

TAC and SOD were measured using the commercial colorimetric reagent kits TAS and RANSOD (Randox Laboratories Ltd., Crumlin, UK) while saliva UA levels were measured using the enzymatic uricase method using commercially available reagents from Roche Diagnostics (Mannheim, Germany). The total protein concentration was determined using Roche Diagnostics' automated turbidimetric urinary and cerebrospinal fluid total protein assay; this measuring range $(0.02-2 \mathrm{~g} / \mathrm{L})$ proved to have satisfactory analytical performance for the measurement of total proteins in the saliva samples. Measurements of TAC, SOD, UA, and total proteins were performed on a Cobas c501 automated biochemistry analyzer (Roche Diagnostics, Mannheim, Germany). All OS markers' values were normalized to the total protein concentration.

For the assays, the analytical performance (including intra- and inter-assay variability) was assessed and is available in our previously published study [28].

\section{Analysis of SC}

Free SC was determined using a commercially available enzyme-linked immunosorbent assay (ELISA) (Demeditec Diagnostics $\mathrm{GmbH}$, Germany) according to the manufacturers' recommendations. This assay measures free SC based on the principle of competitive binding of cortisol from samples and a cortisol-enzyme conjugate to a polyclonal antibody that is pre-coated onto the microtiter wells. The resultant binding is measured spectrophotometrically and is inversely proportional to the concentration of free $\mathrm{SC}$ in the tested samples. The intra- and inter-assay variabilities of this assay kit are 5.8 and $6.4 \%$, respectively, as stated by the manufacturer.

\section{Statistical analysis}

Data analyses were performed using the Statistica 13.4.0 software package (1984-2018 TIBCO Software Inc.) with the alpha set to $p<0.05$. Data distribution was tested using the Shapiro-Wilk test. Before performing the statistical analyses, a log transformation was used for all data that were non-normally distributed (OS markers and SC).

The Student's t-test was used to examine differences upon admission between the TMD subgroups. The changes in the means of measured variables were analyzed using repeated measures analysis of variance (ANOVA) with time (baseline, 1st month, and 3rd months of therapy) as the within factor and pain intensity (HIP and LIP) and source of pain (MP and DD) as 
Table 1 Pretreatment characteristics of the sample

\begin{tabular}{|c|c|c|c|c|}
\hline \multirow[t]{2}{*}{ Variable } & \multicolumn{2}{|c|}{ completed $(n=12)$} & \multicolumn{2}{|c|}{ dropped out $(n=3)$} \\
\hline & mean (SD) & $(95 \% \mathrm{Cl})$ & mean (SD) & $(95 \% \mathrm{Cl})$ \\
\hline Maximal comfortable mouth opening (mm) & $28.00(10.81)$ & $21.13-34.86$ & $31.66(20.21)$ & $-18.53-81.86$ \\
\hline OHIP-14 & $28.22(6.06)$ & $24.47-32.18$ & $19.66(6.02)$ & $4.69-34.63$ \\
\hline PSS & $16.91(6.78)$ & $12.60-21.22$ & $19.33(2.08)$ & $14.16-24.50$ \\
\hline VAS & $70.00(20.33)$ & $51.51-84.83$ & $50.00(10.00)$ & $25.15-74.84$ \\
\hline CPI & $51.07(18.77)$ & $39.14-63.01$ & $39.33(17.01)$ & $-2.29-81.58$ \\
\hline PHQ-15 & $6.25(3.04)$ & $4.31-8.18$ & $11.00(3.61)$ & 2.04-19.95 \\
\hline PHQ-9 & $5.00(4.31)$ & $2.26-7.73$ & $8.00(5.56)$ & $-5.83-21.83$ \\
\hline GAD-7 & $5.58(5.05)$ & $2.37-8.79$ & $7.33(5.85)$ & $-7.22-21.88$ \\
\hline $\mathrm{OBC}$ & $25.16(11.06)$ & $18.13-32.19$ & $33.33(9.86)$ & $8.82-57.84$ \\
\hline
\end{tabular}

PSS Perceived Stress Scale, VAS Visual Analogue Scale, OHIP-14 Oral Health Impact Profile

$C P I$ Characteristic pain intensity: low intensity pain $<50$; high intensity pain $\geq 50$

PHQ-15 physical symptoms: scores of 5,10 , and 15 represent cut-points for low, medium, and high physical symptoms, respectively

PHQ-9 depression: scores of 5, 10, 15, and 20 represent cut-points for mild, moderate, moderately severe, and severe depression

GAD-7 anxiety: scores of 5, 10, and 15 represent cut-points for mild, moderate, and severe anxiety

$O B C$ oral behaviors checklist: 0-16 -represents normal behaviors; 17-24-occurs twice as often in TMD patients; 25-62- risk for TMD

the between factors. Bonferroni's post hoc test was used to show where the differences occurred. Pearson's correlation was used to evaluate the association between changes in SC, the level of perceived stress and OS markers in the TMD subgroups.

\section{Results}

The baseline characteristics of the patients are listed in Table 1.

No significant age differences in the TMD subgroups in terms of pain source (MP $38.33 \pm 10.34$; DD $43.00 \pm 11.13$; $\mathrm{t}=-0.75, p=0.46$ ) or pain intensity (HIP $42.16 \pm 13.71$; LIP $39.16 \pm 7.11 ; \mathrm{t}=0.47, p=0.64$ ) were found.

The mean CPI of the MP subjects $(48.6 \pm 13.36)$ and DD subjects $(53.55 \pm 24.12)$ was not statistically different $(\mathrm{t}=-0.43, p=0.66)$. LIP and HIP were equally distributed among the patients diagnosed with MP and with DD. Three MP subjects and 3 DD subjects had LIP (38.6 and 38.6, respectively). Similarly, $3 \mathrm{MP}$ subjects and 3 DD subjects had HIP (58.53 and 68.43, respectively). However, no significant differences among the groups were found $(p>0.05)$. Therefore, patients were pooled into pain intensity subgroups, regardless of the source of pain. Similarly, patients were pooled into pain source subgroups, regardless of the pain intensity.

\section{Changes in OS markers observed during treatment}

Table 2 lists the mean concentrations of SC as well concentrations of salivary TAC, MDA, SOD, and UA

Table 2 Salivary OS biomarkers and cortisol concentrations in TMD patients and in specific TMD subgroups

\begin{tabular}{|c|c|c|c|c|c|c|c|}
\hline \multirow[t]{3}{*}{ Biomarker } & \multirow{3}{*}{$\begin{array}{l}\text { time } \\
\text { of } \\
\text { day }\end{array}$} & \multicolumn{2}{|c|}{ TMD patients $(n=12)$} & \multicolumn{2}{|c|}{ Diagnosis (source) } & \multicolumn{2}{|l|}{ Pain intensity } \\
\hline & & & & \multirow{2}{*}{$\begin{array}{l}\text { MP }\left(n=6^{*}\right) \\
\text { Mean }\end{array}$} & \multirow{2}{*}{$\begin{array}{l}\mathrm{DD}\left(n=6^{*}\right) \\
\text { Mean }\end{array}$} & \multirow{2}{*}{$\begin{array}{l}\operatorname{Low}\left(n=6^{* *}\right) \\
\text { Mean }\end{array}$} & \multirow{2}{*}{$\begin{array}{l}\text { High }\left(n=6^{* *}\right) \\
\text { Mean }\end{array}$} \\
\hline & & Mean & $(95 \% \mathrm{Cl})$ & & & & \\
\hline \multirow{2}{*}{$\begin{array}{l}\text { TAC }^{+} \\
(\mathrm{mmol} / \mathrm{g})\end{array}$} & AM & 4.99 & $2.1-7.9$ & 5.70 & 4.29 & 2.79 & 7.21 \\
\hline & PM & 4.48 & $2.6-6.3$ & 4.82 & 4.13 & 3.08 & 5.87 \\
\hline \multirow{2}{*}{$\begin{array}{l}\mathrm{MDA}^{+} \\
(\mathrm{nmol} / \mathrm{g})\end{array}$} & AM & 370.54 & $130.8-610.3$ & 466.49 & 274.60 & 222.88 & 518.21 \\
\hline & PM & 761.47 & $368.7-1154.2$ & 750.69 & 772.24 & 498.24 & 1024.69 \\
\hline \multirow{2}{*}{$\begin{array}{l}\mathrm{SOD}^{+} \\
(\mathrm{U} / \mathrm{g})\end{array}$} & AM & 4408.31 & $2583.4-6233.2$ & 3976.86 & 4839.77 & 3739.95 & 5076.68 \\
\hline & PM & 3388.72 & 1882.2-4895.2 & 3006.02 & 3771.41 & 3238.30 & 3539.13 \\
\hline \multirow{2}{*}{$\begin{array}{l}\mathrm{UA}^{+} \\
(\mu \mathrm{mol} / \mathrm{g})\end{array}$} & AM & 661.72 & $208.1-1115.3$ & 796.86 & 526.58 & 357.73 & 965.71 \\
\hline & PM & 613.07 & 288.9-937.18 & 687.96 & 538.19 & 402.90 & 823.25 \\
\hline \multirow[t]{2}{*}{$\mathrm{SC}(\mathrm{ng} / \mathrm{ml})$} & AM & 11.25 & $7.8-14.7$ & 15.40 & 7.10 & 11.68 & 10.82 \\
\hline & PM & 3.09 & $1.8-4.4$ & 3.75 & 2.43 & 3.77 & 2.42 \\
\hline
\end{tabular}

${ }^{+}$Concentrations Protein corrected

*pooled regardless of pain intensity; **pooled regardless of pathology source (diagnostic subgroup)

TAC Total antioxidant capacity, MDA Malondialdehyde, SOD Superoxide dismutase, UA Uric acid, SC Salivary cortisol, CI Confidence interval, TMD

Temporomandibular disorders, DD Disc displacement, MP Myofascial pain 
corrected to total protein concentration for all TMD patients as well as for diagnostic subgroups.

The changes in the TMD subgroups' salivary TAC, MDA, SOD, UA, and SC levels between baseline and after 1 and 3 months of treatment are presented in Table 3. Concentration is expressed in log 10 scale.

The overall afternoon TAC did not differ between TMD subgroups (source of pain: $F=0.09 ; p=0.076$; pain intensity: $F=2.11, p=0.176$ ). but changed significantly over time
(Table 3). The post hoc analysis showed that the mean afternoon TAC was significantly lower at the third month compared to the baseline (source of pain: $p=0.022$; pain intensity: $p=0.033$ ). A decrease of morning TAC was observed in all TMD subgroups. However, the change was not statistically significant.

When evaluating afternoon MDA levels, the analysis revealed a significant interaction between time and pain intensity (Wilks's lambda $=0.42, \mathrm{~F}(2,9)=6.12, p=0.021$,

Table 3 Changes observed in the TMD subgroups during 3 months of occlusal split treatment

\begin{tabular}{|c|c|c|c|c|c|c|c|c|c|c|c|c|c|c|}
\hline & \multirow[t]{3}{*}{ Biomarker $^{a}$} & & \multicolumn{4}{|c|}{ Diagnosis (source) } & \multirow{3}{*}{$\begin{array}{l}p^{+} \\
\text {(TIME) }\end{array}$} & \multicolumn{5}{|c|}{ Pain intensity } & & \\
\hline & & & \multicolumn{2}{|c|}{$\mathrm{MP}\left(n=6^{*}\right)$} & \multicolumn{2}{|c|}{$\mathrm{DD}\left(n=6^{*}\right)$} & & \multicolumn{2}{|c|}{$\operatorname{Low}\left(n=6^{* *}\right)$} & \multicolumn{3}{|c|}{$\operatorname{High}\left(n=6^{* *}\right)$} & \multicolumn{2}{|c|}{ INTERACTION } \\
\hline & & & Mean & $(95 \% \mathrm{Cl})$ & Mean & $(95 \% \mathrm{Cl})$ & & Mean & $(95 \% \mathrm{Cl})$ & Mean & $(95 \% \mathrm{Cl})$ & $\begin{array}{l}\mathrm{p}^{+} \\
\text {(TIME) }\end{array}$ & $\begin{array}{l}\mathrm{p}^{+} \\
\left(\mathrm{TIME} \mathrm{N}^{*}\right. \\
\text { SOURCE) }\end{array}$ & $\begin{array}{l}\mathrm{p}^{+} \\
\text {(TIME* } \\
\text { INTENSITY) }\end{array}$ \\
\hline \multirow[t]{15}{*}{ Morning } & TAC & T0 & 0.62 & $0.25-0.99$ & 0.50 & $0.13-0.87$ & 0.074 & 0.40 & $0.19-0.61$ & 0.72 & $0.31-1.13$ & 0.076 & 0.48 & 0.91 \\
\hline & & $\mathrm{T} 1$ & 0.48 & $0.24-0.71$ & 0.49 & $0.10-0.87$ & & 0.33 & $0.08-0.57$ & 0.63 & $0.35-0.92$ & & & \\
\hline & & $\mathrm{T} 2$ & 0.37 & $0.04-0.70$ & 0.34 & $-0.05-0.74$ & & 0.22 & $-0.02-0.48$ & 0.48 & $0.09-0.48$ & & & \\
\hline & MDA & TO & 2.37 & $1.69-3.05$ & 2.23 & $1.73-2.73$ & 0.15 & 2.09 & $1.54-2.65$ & 2.50 & $1.94-3.06$ & 0.21 & 0.14 & 0.33 \\
\hline & & $\mathrm{T} 1$ & 1.57 & $0.88-2.25$ & 2.07 & $1.33-2.82$ & & 1.75 & $1.13-2.37$ & 1.89 & $1.01-2.78$ & & & \\
\hline & & $\mathrm{T} 2$ & 1.81 & $1.27-2.34$ & 2.28 & $1.75-2.81$ & & 2.07 & $1.61-2.53$ & 2.01 & $1.31-2.71$ & & & \\
\hline & SOD & TO & 3.42 & $2.92-3.91$ & 3.60 & $3.28-3.93$ & 0.51 & 3.43 & $2.99-3.87$ & 3.58 & $3.17-4.01$ & 0.53 & 0.63 & 0.78 \\
\hline & & $\mathrm{T} 1$ & 3.44 & $3.15-3.73$ & 3.48 & $3.16-3.81$ & & 3.35 & $3.12-3.58$ & 3.58 & $3.25-3.90$ & & & \\
\hline & & $\mathrm{T} 2$ & 3.40 & $3.03-3.77$ & 3.39 & $3.07-3.71$ & & 3.31 & $3.10-3.52$ & 3.48 & $3.06-3.91$ & & & \\
\hline & UA & TO & 2.74 & $2.37-3.11$ & 2.60 & $2.22-2.98$ & 0.17 & 2.53 & $2.37-2.69$ & 2.81 & $2.34-3.27$ & 0.19 & 0.35 & 0.98 \\
\hline & & $\mathrm{T} 1$ & 2.60 & $2.43-2.78$ & 2.64 & $2.20-3.08$ & & 2.49 & $2.22-2.76$ & 2.75 & $2.42-3.08$ & & & \\
\hline & & $\mathrm{T} 2$ & 2.50 & $1.96-3.03$ & 2.49 & $1.92-3.07$ & & 2.35 & $1.97-2.73$ & 2.64 & $2.01-3.29$ & & & \\
\hline & SC & TO & 1.17 & $1.06-1.29$ & 0.82 & $0.66-0.98$ & 0.50 & 1.02 & $0.78-1.26$ & 0.97 & $0.72-1.23$ & 0.49 & 0.10 & 0.73 \\
\hline & & $\mathrm{T} 1$ & 1.02 & $0.78-1.24$ & 0.82 & $0.69-0.95$ & & 0.95 & $0.71-1.19$ & 0.88 & $0.70-1.07$ & & & \\
\hline & & $\mathrm{T} 2$ & 1.05 & $0.84-1.26$ & 1.00 & $0.66-1.33$ & & 0.99 & $0.72-1.26$ & 1.06 & $0.77-1.34$ & & & \\
\hline \multirow[t]{15}{*}{ Afternoon } & TAC & TO & 0.60 & $0.34-0.86$ & 0.58 & $0.39-0.77$ & $0.036^{*}$ & 0.48 & $0.38-0.57$ & 0.71 & $0.46-0.95$ & $0.045^{*}$ & 0.17 & 0.50 \\
\hline & & $\mathrm{T} 1$ & 0.50 & $0.26-0.74$ & 0.47 & $0.10-0.84$ & & 0.43 & $0.22-0.63$ & 0.54 & $0.17-0.92$ & & & \\
\hline & & T2 & 0.35 & $0.24-0.46$ & 0.51 & $0.31-0.71$ & & 0.37 & $0.23-0.51$ & 0.49 & $0.29-0.69$ & & & \\
\hline & MDA & TO & 2.76 & $2.39-3.13$ & 2.55 & $1.81-3.30$ & 0.52 & 2.47 & $1.87-3.07$ & 2.84 & $2.31-3.36$ & 0.38 & 0.43 & $0.02^{*}$ \\
\hline & & $\mathrm{T} 1$ & 2.34 & $1.58-3.10$ & 2.69 & $1.87-3.51$ & & 2.86 & $2.18-3.54$ & 2.17 & $1.42-2.92$ & & & \\
\hline & & $\mathrm{T} 2$ & 2.31 & $1.63-2.98$ & 2.53 & $1.82-3.24$ & & 2.62 & $2.49-2.75$ & 2.21 & $1.28-3.14$ & & & \\
\hline & SOD & TO & 3.31 & $2.85-3.76$ & 3.52 & $3.28-3.77$ & 0.23 & 3.40 & $3.01-3.80$ & 3.43 & $3.06-3.80$ & 0.22 & 0.88 & 0.78 \\
\hline & & $\mathrm{T} 1$ & 3.23 & $2.72-3.74$ & 3.41 & $3.24-3.58$ & & 3.27 & $2.81-3.73$ & 3.37 & $3.06-3.68$ & & & \\
\hline & & $\mathrm{T} 2$ & 3.29 & $3.03-3.55$ & 3.54 & $3.38-3.71$ & & 3.41 & $3.13-3.68$ & 3.43 & $3.18-3.67$ & & & \\
\hline & UA & TO & 2.69 & $2.31-3.07$ & 2.70 & $2.52-2.88$ & 0.39 & 2.56 & $2.34-2.78$ & 2.83 & $2.55-3.11$ & 0.30 & 0.76 & $0.03^{*}$ \\
\hline & & $\mathrm{T} 1$ & 2.68 & $2.37-2.99$ & 2.74 & $2.51-2.97$ & & 2.69 & $2.45-2.92$ & 2.73 & $2.43-3.04$ & & & \\
\hline & & $\mathrm{T} 2$ & 2.56 & $2.19-2.93$ & 2.67 & $2.33-3.01$ & & 2.51 & $2.23-2.77$ & 2.73 & $2.34-3.12$ & & & \\
\hline & SC & TO & 0.50 & $0.19-0.80$ & 0.28 & $0.04-0.60$ & 0.99 & 0.50 & $0.20-0.80$ & 0.28 & $0.04-0.60$ & 0.99 & 0.24 & $0.04^{*}$ \\
\hline & & $\mathrm{T} 1$ & 0.45 & $0.07-0.82$ & 0.33 & $0.11-0.55$ & & 0.41 & $0.15-0.69$ & 0.35 & $0.01-0.70$ & & & \\
\hline & & $\mathrm{T} 2$ & 1.57 & $0.17-0.98$ & 0.21 & $0.05-0.38$ & & 0.30 & $0.08-0.53$ & 0.48 & $0.03-0.93$ & & & \\
\hline
\end{tabular}

*pooled regardless of pain intensity; **pooled regardless of pathology source (diagnostic subgroup)

TAC Total antioxidant capacity, MDA Malondialdehyde, SOD Superoxide dismutase, UA Uric acid, SC Salivary cortisol, CI Confidence interval, TMD

Temporomandibular disorders, DD Disc displacement, MP Myofascial pain, TO baseline, $T 1$ 1st month of treatment, T2 3rd month of treatment

${ }^{a} \log$ transformed data; ${ }^{+}$analysis of variance (ANOVA) for repeated measurements 
Table 4 Means and SD of log biomarker ratio in TMD subgroups

\begin{tabular}{|c|c|c|c|c|c|c|c|c|}
\hline & \multicolumn{2}{|l|}{ Log biomarker ratio } & \multicolumn{2}{|c|}{ Diagnosis (source) } & \multicolumn{2}{|l|}{ Pain intensity } & \multirow{2}{*}{$\begin{array}{l}\mathrm{p} \\
\text { (TIME*SOURCE) }\end{array}$} & \multirow{2}{*}{$\begin{array}{l}\mathrm{p} \\
\text { (TIME*INTENSITY }\end{array}$} \\
\hline & & & $\mathrm{MP}\left(n=6^{*}\right)$ & $\mathrm{DD}\left(n=6^{*}\right)$ & $\operatorname{Low}\left(n=6^{* *}\right)$ & $\operatorname{High}\left(n=6^{* *}\right)$ & & \\
\hline \multirow[t]{3}{*}{ Morning } & MDA/SOD, Mean (SD) & TO & $-1.04(0.73)$ & $-1.37(0.43)$ & $-1.34(0.80)$ & $-1.08(0.33)$ & 0.12 & 0.56 \\
\hline & & $\mathrm{T} 1$ & $-1.87(0.55)$ & $-1.41(0.59)$ & $-1.59(0.68)$ & $-1.68(0.57)$ & & \\
\hline & & $\mathrm{T} 2$ & $-1.61(0.65)$ & $-1.11(0.36)$ & $-1.24(0.58)$ & $-1.46(0.57)$ & & \\
\hline \multirow[t]{3}{*}{ Afternoon } & MDA/SOD, Mean (SD) & TO & $-0.54(0.36)$ & $-0.97(0.74)$ & $-0.92(0.80)$ & $-0.59(0.29)$ & 0.42 & 0.02 \\
\hline & & $\mathrm{T} 1$ & $-0.89(0.84)$ & $-0.71(0,78)$ & $-0.41(0,71)$ & $-1.19(0.68)$ & & \\
\hline & & $\mathrm{T} 2$ & $-0.98(0.61)$ & $-1.01(0.63)$ & $-0.78(0.28)$ & $-1.21(0.76)$ & & \\
\hline
\end{tabular}

*pooled regardless of pain intensity; **pooled regardless of pathology source (diagnostic subgroup)

MDA Malondialdehyde, SOD Superoxide dismutase, DD Disc displacement, MP Myofascial pain, SD Standard deviation, TO baseline, $T 1$ st month of treatment, $T 2$ 3rd month of treatment

$\left.\eta_{\mathrm{p}}{ }^{2}=0.33\right)$. HIP patients showed a significant reduction in afternoon MDA at the first and third month of treatment compared to the baseline $(p=0.012$ and $p=0.017$, respectively) while in LIP patients the levels of afternoon MDA remained unchanged. A greater reduction in afternoon
MDA levels during the therapy period was observed in the MP group when compared to DD patients, but the decrease was not statistically significant.

A greater reduction in morning MDA was observed in HIP patients when compared to LIP patients, as well as
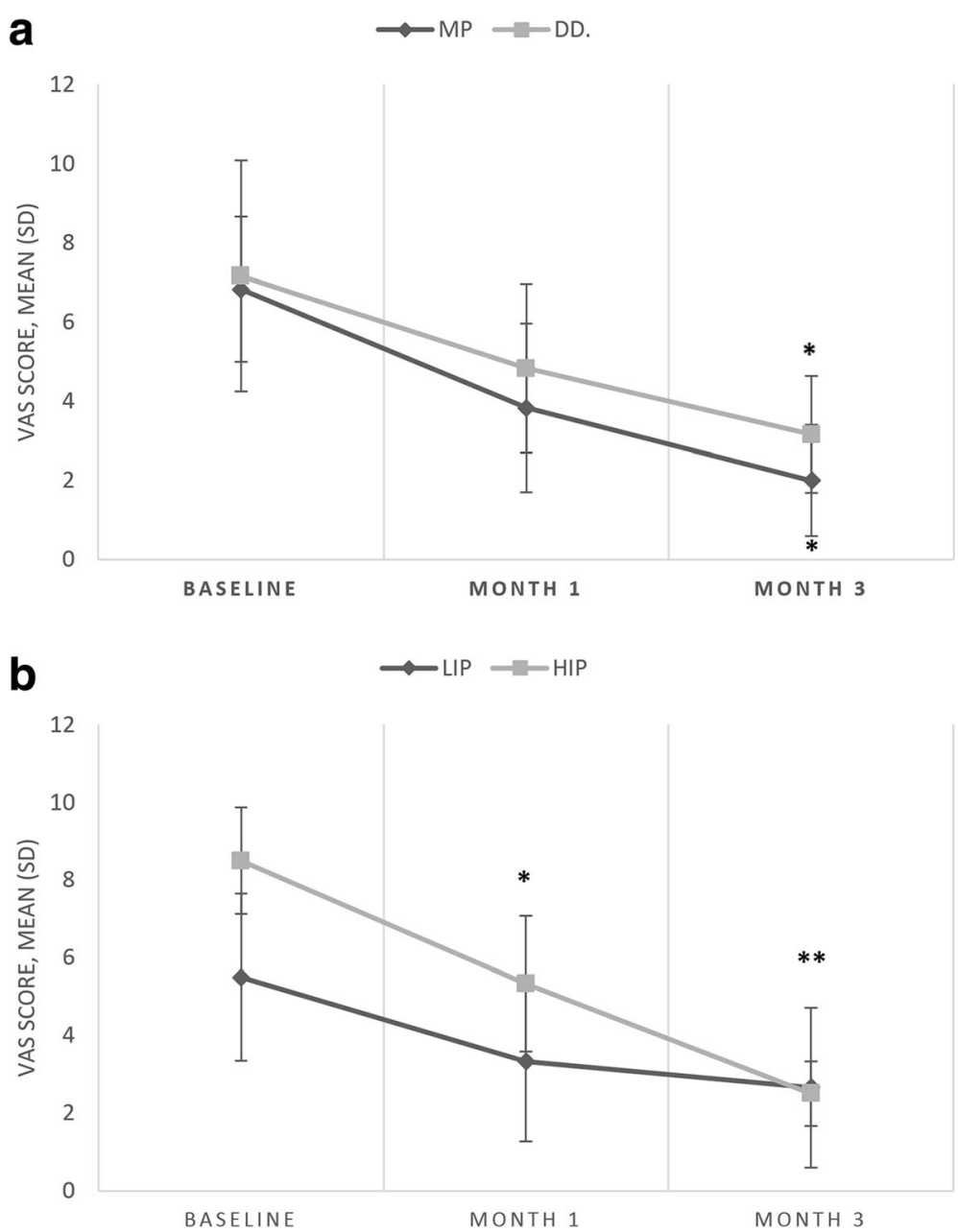

Fig. 2 ab Visual analogue scale scores (means and standard deviations) during the 3-month interval of occlusal splint therapy. Asterisk indicates a post-hoc significant difference (within group comparisons, compared to baseline). ${ }^{*} p<0.05{ }^{* *} p<0.001$. VAS, Visual Analogue Scale; MP, myofascial pain; DD, disc displacement; HIP, high-intensity pain; LIP, low-intensity pain 
in MP patients when compared to DD patients, but the difference was not statistically significant (pain intensity $F=0.38, p=0.55$; source of pain: $F=1.24, p=0.29)$ ).

The analysis revealed a significant interaction between time and pain intensity in terms of afternoon UA (Wilks's lambda $=0.46, \mathrm{~F}(2,9)=5.11, p=0.032, \eta_{\mathrm{p}}{ }^{2}=$ 0.15). A significant reduction in afternoon UA in LIP patients was observed in the third month of treatment compared to the baseline. In HIP patients, the decrease in afternoon UA was not statistically significant.

Morning SC did not change significantly over time, but differed significantly between the MP and DD subgroups $(F=6.38, p=0.03)$ with higher values in MP patients at all-time points. The effect of treatment on afternoon SC differed significantly between high and low intensity pain subgroups (Wilks's lambda $=0.48, F(2,9)$ $\left.=4.71, p=0.039, \eta_{\mathrm{p}}{ }^{2}=0.34\right)$. Post hoc tests revealed that afternoon SC decreased significantly in LIP patients and increased in HIP patients.

When evaluating afternoon MDA to SOD ratios (Table 4), the repeated measures ANOVA revealed a significant interaction between time and pain intensity (Wilks's lambda $=$ $0.40, \mathrm{~F}(2,9)=7.18, p=0.017, \eta_{\mathrm{p}}{ }^{2}=0.30$ ). Afternoon MDA to SOD ratios decreased significantly in HIP patients at the first and third month of treatment compared to the baseline ( $p=0.039$ and $p=0.034$, respectively) while in LIP patients, the MDA to SOD ratios remained unchanged at the end of therapy when compared to baseline levels.

\section{Changes in VAS, OHIP-14, PSS, and MCO}

When considering the source of pain, VAS scores did not differ between TMD subgroups $(F=1.07 ; p=0.032)$, but changed significantly over time (Wilks's lambda $=0.25$, F (2, 9) $=13.5, p=0.002, \eta_{p}^{2}=0.63$ ) (Fig. 2). VAS scores were,

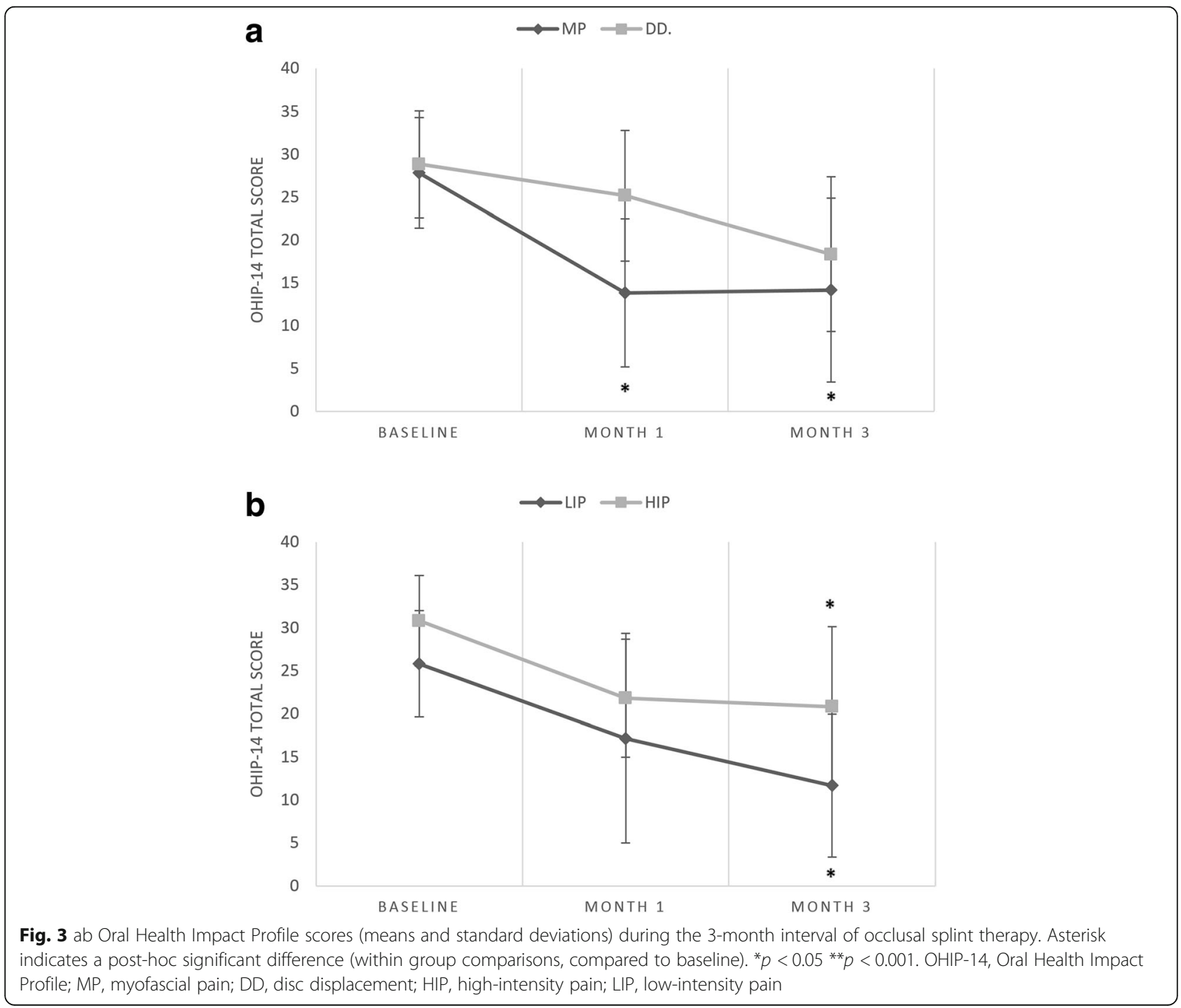


however, higher in HIP patients when compared to LIP patients $(F=5.71, p=0.038)$ and were significantly influenced by time (Wilks's lambda $=0.21, \mathrm{~F}(2,9)=16.48, p=0.001$, $\left.\eta_{\mathrm{p}}^{2}=0.68\right)$.

Oral health-related quality of life improved significantly over time in HIP and LIP patients (Wilks's lambda $\left.=0.37, \mathrm{~F}(2,9)=7.61, p=0.011, \eta_{\mathrm{p}}{ }^{2}=0.53\right)$, but did not differ between groups $(F=2.79, p=0.13)$. Significant improvement in OHIP-14 scores during the treatment period was observed both in MP and DD patients (Wilks's lambda $=0.34, \mathrm{~F}(2,9)=8.36, p=0.008$, $\eta_{\mathrm{p}}{ }^{2}=0.57$ ). The effect of treatment on OHIP-14 scores was more pronounced in MP patients (Wilks's lambda = 0.51, $\mathrm{F}(2,9)=4.29, p=0.048, \eta_{\mathrm{p}}{ }^{2}=0.19$ ). (Fig. 3).

Pain-free MCO increased over time and differed significantly between the MP and DD subgroups $(F=5.01$, $p=0.049$ ) (Fig. 4). At all time points, values were higher in MP patients.
A decrease in the levels of perceived stress was seen only in the MP group, but the changes were not statistically significant $(p>0.05)$ (Fig. 5).

\section{Association between PSS, SC, and OS markers}

At baseline, higher levels of perceived stress were significantly associated with higher morning cortisol $(\rho=0.67$, $p=0.016$ ). However, the association between perceived stress and SC with OS markers was not significant when considering all TMD patients. When correlations were tested separately for the TMD subgroups, a significant positive correlation between morning cortisol and OS markers was found in MP patients (Table 5).

At the end of the therapy, the percentage change in morning SC showed a significant positive correlation with reduced perceived stress $(\rho=0.58, p=0.048)$ when all TMD patients were considered.

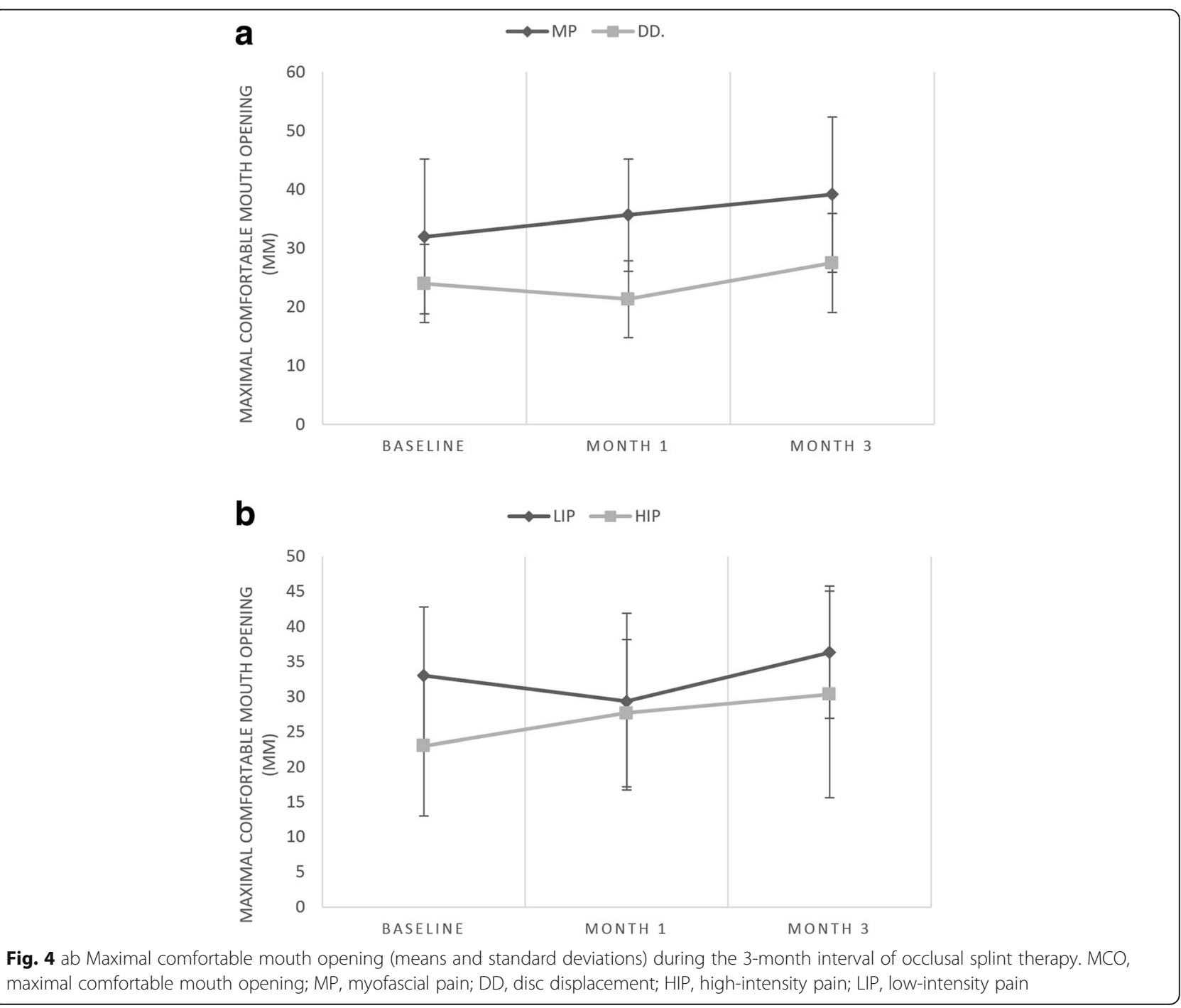



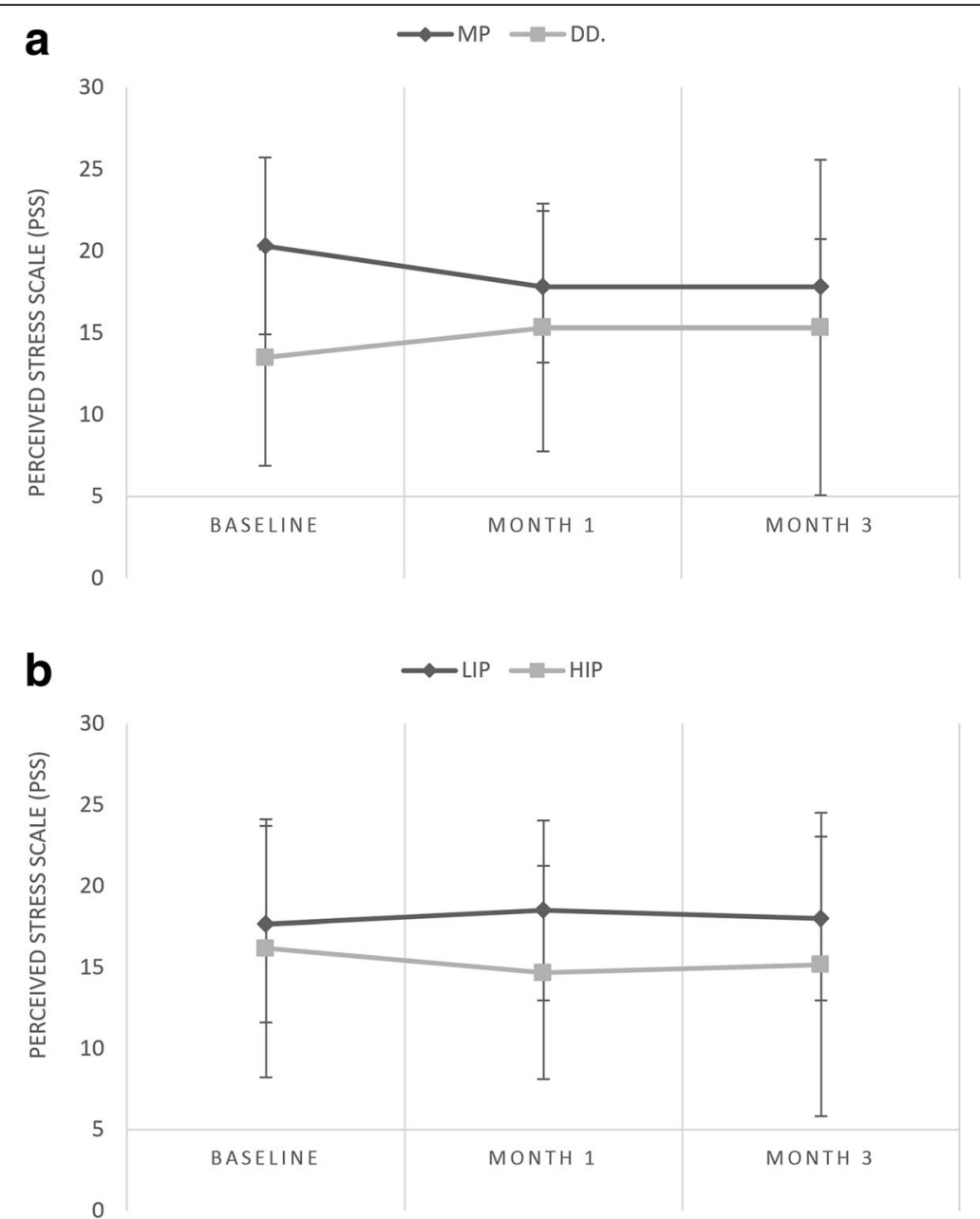

Fig. 5 ab Change in perceived stress level (means and standard deviations) during the 3-month interval of occlusal splint therapy. PSS, Perceived Stress Scale; MP, myofascial pain; DD, disc displacement; HIP, high-intensity pain; LIP, low-intensity pain

When correlations were tested separately for the TMD subgroups, a significant positive correlation between reduced perceived stress and the percentage change in morning TAC $(\rho=0.86, p=0.025)$ was observed in MP patients.

\section{Discussion}

When it comes to TAC levels in various diseases, data are inconsistent. Research addressing oxidative status in TMD patients is scarce $[17,18,29-31]$, and to the best of our knowledge, no study has attempted to track changes in OS biomarker levels during TMD therapy. Rodriguez de Sotillo et al. and De Almeida and Amenábar found that TAC levels were lower in the TMD group than in control groups, indicating reduced antioxidant protection [17, 31]. However, our previous research found TAC levels to be higher in the TMD group compared to the control group. The main reason for these differences may be that in our research, we chose only female patients with chronic pain lasting 6 months or more. This six-month period provided an opportunity for the patients to adjust to potentially high oxidant levels, resulting in higher TAC levels [18]. Similar opposing results, where researchers observed higher or lower levels of TAC, have been found in other pathologies as well (e.g., atherosclerosis) [32, 33].

The higher levels of TAC previously found in TMD patients were explained as a compensatory reaction to a distorted oxidative balance [18]; therefore, the decrease in TAC levels following the drop in oxidant levels during occlusal splint therapy was expected. The decrease in TAC over time was followed by a significant reduction in the MDA levels of the HIP group. This may be due to the diminished need for antioxidative defense after the decrease in pro-oxidant activity.

Researchers have pointed out wide variability as a problem when assaying the antioxidant biomarker MDA [34]. Because of the variability in OS markers, future 
Table 5 Correlations among psychological stress, cortisol reactivity, and oxidative stress markers in TMD patients

\begin{tabular}{|c|c|c|c|c|c|c|c|c|}
\hline & & & & PSS & TAC & MDA & SOD & UA \\
\hline \multirow[t]{3}{*}{ TMD patients $(n=12)$} & & PSS & & - & 282 & ,076 &, 256 & 098 \\
\hline & & SC & morning &, $673^{*}$ &, 264 & 280 & ,086 & ,305 \\
\hline & & & afternoon &, 216 &,- 252 &, 225 & ,198 & , 106 \\
\hline \multirow[t]{6}{*}{ Diagnosis (source) pooled regardless of pain intensity } & $\mathrm{MP}(n=6)$ & PSS & & - &, $721^{*}$ & ,399 &, $780^{*}$ & ,305 \\
\hline & & SC & morning & ,795* &, $781^{*}$ &, 064 &, $958^{*+}$ & ,271 \\
\hline & & & afternoon & ,136 &,- 028 &, 512 & ,605 & ,226 \\
\hline & $\mathrm{DD}(n=6)$ & PSS & & - &,- 191 &,- 422 &, 131 &,- 260 \\
\hline & & SC & morning & ,327 &,- 070 &, 563 &, 130 & ,231 \\
\hline & & & afternoon & 234 &,- 639 &, 017 &, 003 &,- 049 \\
\hline \multirow[t]{6}{*}{ Pain intensity pooled regardless of pathology source } & Low $(n=6)$ & PSS & & - & ,622 &,- 032 &, 521 &,- 276 \\
\hline & & SC & morning &, 535 & ,333 &,- 031 &,- 038 & ,267 \\
\hline & & & afternoon &,- 237 &,- 041 &, 297 & ,219 & ,631 \\
\hline & High $(n=6)$ & PSS & & - & ,311 &, 258 & , 102 & ,285 \\
\hline & & SC & morning &, $773^{*}$ & ,394 & ,699 & ,255 &, 469 \\
\hline & & & afternoon &, $731^{*}$ &,- 044 &, 543 & ,248 & 145 \\
\hline
\end{tabular}

PSS Perceived Stress Scale, TAC Total antioxidant capacity, MDA Malondialdehyde, SOD Superoxide dismutase, UA Uric acid, SC Salivary cortisol

"Note: the association was significant $p<0.05$

${ }^{+}$Note: the significant association was found also between morning SC and afternoon SOD $(\rho=0.79, p<0.05)$

research will require a larger number of participants to obtain statistical significance. Nevertheless, it is important to note that MDA and the MDA to SOD ratio showed a significant decrease in the HIP group, suggesting that in this case, antioxidant biomarkers prevail over oxidants, meaning that HIP in female patients might have a stronger response to occlusal splint therapy.

UA is often mentioned as a paradoxical biomarker because it can have both antioxidant and oxidant characteristics [35]. Our study showed a significant decrease at levels similar to TAC, indicating that in this study, UA might indicate antioxidant activity. Because concentrations of salivary UA linearly follow UA levels in plasma [36, 37], and because studies suggest that UA is the antioxidant in blood [35], these results are justified.

The reduction in morning SC levels was more prominent in the MP patients when compared to the DD patients. This change in morning SC levels and the correlation between morning SC and perceived stress with OS markers observed in the MP patients implies that stress responses may have a larger impact on female patients with pain of myofascial origin. MP is often related to enhanced stress reactivity, and coping with chronic pain may lead to a larger stress response [38]. Moreover, the frequent experience of stress and anxiety may lead to the development of painful trigger points in muscles resulting in MP [39]. The minor influence of cortisol in the DD patients may be due to the fact that stress is less of a factor in disorders connected with the alteration of anatomical structures, but it surely cannot be excluded as a contributing element.
MCO improved during the treatment period, but the improvement was not statistically significant. We need to take into consideration the clinical importance of these results because an increase of only a few millimeters in MCO can have an enormous impact on a patient's quality of life. In the current study, the MCO's tendency to increase was a clear sign of patients' improvement in TMD symptomatology, and it was naturally followed by an improvement in patients' oral healthrelated quality of life. The significant difference observed between MP and DD patients showed that, in terms of the MCO, female MP patients had a better response to occlusal splint therapy. Research shows that there is probably no possibility of restoring the disc to its natural position, especially in chronic DD TMD [40]; hence, the altered anatomy of the joint, i.e., the biological barrier (displaced disc) that is blocking the condyle, may be the reason for a weaker MCO-related response to occlusal splint therapy.

There were a few limitations to this study. One of these was the small sample size, especially when considering the variability of the tested biomarkers. With a larger number of participants, more significant differences would most certainly occur. Second, our subjects were exclusively women; this was because few men came to the clinic with TMD-associated difficulties, which is in accordance with the fact that women are much more likely to be affected [3]. Because women are two times more likely to be affected with TMD (National Institute of Dental and Craniofacial Research) this limitation is often present in research on TMD. At the time of the 
current study, there are no similar studies, so there was no possibility of comparing the results. In addition, our study lacked a non-treatment or placebo group, so we cannot rule out changes that may have occurred spontaneously. Nevertheless, respondents were monitored for 3 months; thus, the results cannot be disregarded, and we strongly encourage further research on this topic.

\section{Conclusion}

The results of this study, conducted among female, chronic TMD patients, show that during 3 months of occlusal splint therapy, the TAC levels in the patients' saliva significantly decreased. The MDA to SOD ratios decreased, and this reduction was more pronounced in HIP patients. This decrease in the MDA to SOD ratio might indicate that the effect of splint therapy, which may be seen as a reduction of TMD symptoms in female TMD patients, might help in body's cleansing capacity to remove free radicals. The question remains whether or not TAC decreases in this process as a result of avoiding unnecessary processes, once the increase in antioxidants effectively compensates for OS. When considering the association between SC and perceived stress with OS markers, it is important to emphasize that the positive correlation was present in female MP patients. The effect of treatment on the self-perceived quality of life was more pronounced in female MP patients while the reduction of spontaneous pain, although present in all TMD subgroups, was significantly greater in patients with HIP.

Although the study used a small sample size of only women, our results emphasize that both the source and the intensity of pain should be considered in future investigations.

\section{Abbreviations}

ANOVA: Repeated-measurements analysis of variance; CPI: Characteristic pain intensity; DD: Disc displacement; ELISA: Enzyme-linked immunosorbent assay; HIP: High intensity pain; LIP: Low intensity pain; MCO: Maximal comfortable mouth opening, OHIP-14 Oral health impact profile; MDA: Malondialdehyde; MP: Myofascial pain; OHIP: Oral health impact factor; OS: Oxidative stress; PSS: Perceived stress scale; SC: Salivary cortisol; SOD: Superoxide dismutase; TAC: Total antioxidant capacity; TMD: Temporomandibular disorders; UA: Uric acid; VAS: Visual analogue scale
\end{abstract}

\section{Acknowledgements}

Not applicable.

\section{Authors' contributions}

Contributor EV collected the saliva samples, performed clinical examination of patients at baseline and at follow - up appointments and wrote the manuscript. IZA designed the study, analyzed the data, sorted patients into groups and wrote the manuscript. IL measured the samples and co-wrote the manuscript. DR contributed to the concept and design, and measured the samples. All authors read and approved the final manuscript.

\section{Funding}

This research is a part of a project "The Role of Oxidative Stress and Opiorphin in Temporomandibular Disorders" (No. IP-2014-09-3070) supported by the Croatian Science Foundation (CSF), Operational Programme "Efficient
Human Resources 2014-2020", and European Social Fund within the "Young Researchers' Career Development Project - Training of Doctoral Students". CSF provided funds for all the materials needed in the clinical work and work of the biochemical laboratory that did sample analysis. In addition, CSF provided funds for publishing this article. Operational Program "Efficient Human Resources 2014-2020", and the European Social Fund within the "Young Researchers' Career Development Project - Training of Doctoral Students" provided funding for employment and PhD training of the first author (EV). Details are available at http://www.hrzz.hr/default.aspx?id=78\&pid= 2875\&rok=2014-09

\section{Availability of data and materials}

The data and materials generated and analyzed during the current study are not publicly available. However, all data from this study is available from the corresponding author on reasonable request.

\section{Ethics approval and consent to participate}

The study was approved at the University of Zagreb, School of dental medicine by Ethics Committee (01-PA-26-6/15, item 3.2) and regulated in accordance with the Helsinki Declaration. All subjects were informed and provided written consent before being involved in the research.

\section{Consent for publication}

Not applicable.

\section{Competing interests}

The authors declare that they have no competing interests.

\section{Author details}

'Department of Removable Prosthodontics, School of Dental Medicine, University of Zagreb, Gunduliceva 5, HR-10000 Zagreb, Croatia. ${ }^{2}$ Department of Laboratory Diagnostics, University Hospital Center Zagreb, Zagreb HR-10000, Croatia.

Received: 12 February 2019 Accepted: 20 May 2019

Published online: 06 June 2019

\section{References}

1. Gauer RL, Semidey MJ. Diagnosis and treatment of temporomandibular disorders. Am Fam Physician. 2015;91(6):378-86.

2. Schiffman E, Ohrbach R, Truelove E, Look J, Anderson G, Goulet JP, et al. Diagnostic criteria for temporomandibular disorders (DC/TMD) for clinical research applications: recommendations of the international RDC/TMD consortium network and orofacial pain special interest group. J Oral Facial Pain Headache. 2014;28(1):6-27. https://doi.org/10.11607/jop.1151.

3. Warren M, Fried JL. Temporomandibular disorders and hormones in women. Cells Tissues Organs. 2001;169:187-92. https:/doi.org/10.1159/000047881.

4. Wright EF, North SL. Management and treatment of temporomandibular disorders: a clinical perspective. J Man Manip Ther. 2009;17(4):247-54.

5. Al-Ani MZ, Davies SJ, Gray RJ, Sloan P, Glenny AM. Stabilisation splint therapy for temporomandibular pain dysfunction syndrome. Cochrane Database Syst Rev. 2004;1:CD002778.

6. Gomes CA, El Hage Y, Amaral AP, Politti F, Biasotto-Gonzalez DA. Effects of massage therapy and occlusal splint therapy on electromyographic activity and the intensity of signs and symptoms in individuals with temporomandibular disorder and sleep bruxism: a randomized clinical trial. Chiropr Man Therap. 2014;22:43.

7. Michelotti A, lodice G, Vollaro S, Steenks MH, Farella M. Evaluation of the short-term effectiveness of education versus an occlusal splint for the treatment of myofascial pain of the jaw muscles. J Am Dent Assoc. 2012;143:47-53.

8. Kreiner M, Betancor E, Clark GT. Occlusal stabilization appliances. Evidence of their efficacy. J Am Dent Assoc. 2001;132:770-7.

9. Gavish A, Winocur E, Ventura YS, Halachmi M, Gazit E. Effect of stabilization splint therapy on pain during chewing in patients suffering from myofascial pain. J Oral Rehabil. 2002;29(12):1181-6.

10. Weidinger A, Kozlov AV. Biological activities of reactive oxygen and nitrogen species: oxidative stress versus signal transduction. Biomolecules. 2015;5(2):472-84.

11. Li S, Tan HY, Wang N, Zhang-Jin Z, Lao L, Chi-Woon W, Feng Y. The role of oxidative stress and antioxidants in liver diseases. Int J Mol Sci. 2015;16: 26087-124. 
12. Bhattacharyya A, Chattopadhyay R, Mitra S, Crowe SE. Oxidative stress: an essential factor in the pathogenesis of gastrointestinal mucosal diseases. Physiol Rev. 2014;94(2):329-54.

13. Kim GH, Kim JE, Rhie SJ, Yoon S. The role of oxidative stress in neurodegenerative diseases. Exp Neurobiol. 2015;24(4):325-40.

14. Agha-Hosseini F, Mirzaii-Dizgah I, Farmanbar N, Abdollahi M. Oxidative stress status and DNA damage in saliva of human subjects with oral lichen planus and oral squamous cell carcinoma. J Oral Pathol Med. 2012;41(10):736-40.

15. Kumar J, Teoh SL, Das S, Mahakknaukrauh P. Oxidative stress in Oral diseases: understanding its relation with other systemic diseases. Front Physiol. 2017;8:693.

16. Gümüş $P$, Emingil $G$, Öztürk VÖ, Belibasakis GN, Bostanci N. Oxidative stress markers in saliva and periodontal disease status: modulation during pregnancy and postpartum. BMC Infect Dis. 2015;15:261.

17. Rodriguez de Sotillo D, Velly AM, Hadley M, Fricton JR. Evidence of oxidative stress in temporomandibular disorders: a pilot study. J Oral Rehabil. 2011; 38(10):722-8. https://doi.org/10.1111/j.1365-2842.2011.02216.x.

18. Vrbanović E, Alajbeg IZ, Vuletić L, Lapić I, Rogić D, Andabak Rogulj A, Illeš D, Knezović Zlatarić D, Badel T, Alajbeg I. Salivary oxidant/antioxidant status in chronic temporomandibular disorders is dependent on source and intensity of pain - a pilot study. Front Physiol. 2018;9:1405. https://doi.org/10.3389/ fphys.2018.01405.

19. Aschbaher K, O'Donovan A, Wolkowitz OM, Dhabhar FS, Su Y, Epell E. Good stress, bad stress and oxidative stress: insights from anticipatory cortisol reactivity. Psychoneuroendocrinology. 2013;38(9):1698-708.

20. Dickerson S, Kemeny ME. Acute stressors and cortisol responses: a theoretical integration and synthesis of laboratory research. Psychol Bull. 2004;130:355-91

21. Monnier L, Mas E, Ginet C, Michel F, Villon L, Cristol JP, Colette C. Activation of oxidative stress by acute glucose fluctuations compared with sustained chronic hyperglycemia in patients with type 2 diabetes. JAMA. 2006;295:1681-7.

22. Valko M, Rhodes CJ, Moncol J, Izakovic M, Mazur M. Free radicals, metals and antioxidants in oxidative stress-induced cancer. Chem Biol Interact. 2006;160:1-40.

23. Spiers JG, Chen HJ, Sernia C, Lavidis NA. Activation of the hypothalamicpituitary-adrenal stress axis induces cellular oxidative stress. Front Neurosci. 2015:8:456.

24. Emshoff R, Emshoff I, Bertram S. Estimation of clinically important change for visual analog scales measuring chronictemporomandibular disorder pain. J Orofac Pain. 2010 Summer;24(3):262-9.

25. Hudek-Knežević J, Kardum I, Lesić R. Efekti percipiranog stresa i stilova suočavanja na tjelesne simptome. Društvena istraživanja. 1999;8:543-61.

26. Rener-Sitar K, Petričević N, Čelebić A, Marion L. Psychometric properties of Croatian and Slovenian short form of oral health impact profile questionnaires. Croat Med J. 2008;49:536-44.

27. Barros Vde M, Seraidarian PI, Côrtes MI, de Paula LV. The impact of orofacial pain on the quality of life of patients with temporomandibular disorder. J Orofac Pain. 2009 Winter;23(1):28-37.

28. Alajbeg IZ, Lapic I, Rogic D, Vuletic L, Andabak Rogulj A, Illes D, et al. Withinsubject reliability and between-subject variability of oxidative stress markers in saliva of healthy subjects: a longitudinal pilot study. Dis Markers. 2017: 2697464. https://doi.org/10.1155/2017/2697464.

29. Basi DL, Velly AM, Schiffman EL, Lenton PA, Besspiata DA, Rankin AM, et al. Human temporomandibular joint and myofascial pain biochemical profiles: a case-control study. J Oral Rehabil. 2012;39:326-37. https://doi.org/10.1111/ j.1365-2842.2011.02271.x.

30. Etöz OA, Akçay H, Neşelioğlu S, Erel Ö, Alkan A. Total antioxidant capacity and total oxidant status of synovial fluids in patients with temporomandibular joint pain and dysfunction. Clin Oral Investig. 2012;16: 1557-61. https://doi.org/10.1007/s00784-011-0666-0.

31. De Almeida C, Amenábar JM. Changes in the salivary oxidative status in individuals with temporomandibular disorders and pain. J Oral Biol Craniofac Res. 2016;6:S1-4. https://doi.org/10.1016/j.jobcr.2016.10.006.

32. Torac E, Stoian I, Gaman L, Magda S, Vinereanu D, Mincu R, losif L, Atanasiu V. PP40 - Total antioxidant capacity (TEAC) is decreased despite high levels of uric acid in patient with atherosclerosis. Free Radic Biol Med. 2015;86(1):S32-3.

33. Nieto FJ, Iribarren C, Gross MD, Comstock GW, Cutler RG. Uric acid and serum antioxidant capacity: a reaction to atherosclerosis? Atherosclerosis. 2000;148:131-9.
34. Khoubnasabjafari M, Ansarin K, Jouyban A. Salivary malondialdehyde as an oxidative stress biomarker in oral and systemic diseases. J Dent Res Dent Clin Dent Prospects. 2016;10(2):71-4.

35. Sautin $Y Y$, Johnson RJ. Uric acid: the oxidant-antioxidant paradox. Nucleosides Nucleotides Nucleic Acids. 2008;27(6):608-19. https://doi.org/10. 1080/15257770802138558

36. Goll RD, Mookerjee BK. Correlation of biochemical parameters in serum and saliva in chronic azotemic patients and patients on chronic hemodialysis. J Dial. 1978;2:344-99.

37. Nunes $L A$, Brenzikofer $R$, Macedo DV. Reference intervals for saliva analytes collected by a standardized method in a physically active population. Clin Biochem. 2011;44:1440-4. https://doi.org/10.1016/j.clinbiochem.2011.09.012.

38. Davis MC, Zautra AJ, Reich JW. Vulnerability to stress among women in chronic pain from fibromyalgia and osteoarthritis. Ann Behav Med. 2001; 23(3):215-26.

39. Jafri MS. Mechanisms of myofascial pain. Int Sch Res Notices. 2014;2014: 523924.

40. Kirk WS Jr. Magnetic resonance imaging and tomographic evaluation of occlusal appliance treatment for advanced internal derangement of the temporomandibular joint. J Oral Maxillofac Surg. 1991;49(1):9-12.

\section{Publisher's Note}

Springer Nature remains neutral with regard to jurisdictional claims in published maps and institutional affiliations.

\section{Ready to submit your research? Choose BMC and benefit from:}

- fast, convenient online submission

- thorough peer review by experienced researchers in your field

- rapid publication on acceptance

- support for research data, including large and complex data types

- gold Open Access which fosters wider collaboration and increased citations

- maximum visibility for your research: over $100 \mathrm{M}$ website views per year

At BMC, research is always in progress.

Learn more biomedcentral.com/submissions 